\title{
Homogenization of Periodically \\ Perforate Media
}

\section{Conca, D. Gómez, M. Lobo \&3 M. E. Pérez}

\begin{abstract}
We consider Bloch wave decomposition to study the homogenization of periodically heterogeneous and perforate media in a bounded domain $\Omega$ of $\mathbb{R}^{N}$. Assuming that $\varepsilon$ is the size of the periodicity of the structure and of the holes, we study the asymptotic behavior, as $\varepsilon \rightarrow 0$, of the solution of an elliptic boundary value problem with strongly oscillating coefficients posed in $\Omega^{\varepsilon}\left(\Omega^{\varepsilon}\right.$ being $\Omega$ minus the holes) with a homogeneous Dirichlet condition on the boundary of $\Omega$ and a Neumann condition on the boundary of the holes. We first consider the case when $\Omega$ is $\mathbb{R}^{N}$ and then localize the problem for a bounded domain $\Omega$. A new characterization of the homogenized coefficients is given via the Hessian at the origin of the first Bloch eigenvalue.
\end{abstract}

1. Introduction. In this paper we consider a Bloch wave decomposition technique in the study of a homogenization problem posed in a domain containing many small periodically distributed holes. As is known, Bloch wave decomposition provides the spectral resolution for self-adjoint operators associated with differential operators with periodic coefficients and it is the standard tool for transforming partial differential equations with variable periodic coefficients into a set of algebraic equations (see [6], [10], and [11] for example). Bloch expansions give a spectral representation of the solution in terms of the eigenvalues and eigenfunctions of a specific family of eigenvalue problems in the reference cell. This family depends on the so-called Bloch parameter $\eta$ which varies in the dual cell $Y^{\prime}=\left[-\frac{1}{2}, \frac{1}{2}\right)^{N}$.

Historically, in mathematics literature, the study of differential equations with periodic coefficients is known as Floquet theory. In physics, it goes under 
the name of Bloch waves method, because it was F. Bloch (see [7]) who first introduced this technique in his study of the motion of an electron in a crystalline solid.

We deal here with the asymptotic behavior of the solution of a classical homogenization problem. More precisely, we consider a heterogeneous material filling a domain $\Omega$ of $\mathbb{R}^{N}$ which contains a periodically distributed structure of perforations. We assume that the size of the holes and the periodicity depend on a small parameter $\varepsilon$, and both are of the same order of magnitude $O(\varepsilon)$. We denote by $\Omega^{\varepsilon}$ the domain $\Omega$ minus these holes, and study the asymptotic behavior, as $\varepsilon \rightarrow 0$, of the solution of an elliptic boundary value problem, posed in $\Omega^{\varepsilon}$ with a homogeneous Dirichlet condition on the boundary of $\Omega$, and a Neumann condition on the boundary of the holes.

As is well-known in homogenization theory, asymptotic expansions and rigorous mathematical analysis techniques are complementary devices in the analysis of this kind of problem. We use here the Bloch wave decomposition as a self-contained method, to obtain the homogenized problem as well as to prove convergence of the solutions. We show that this method obviates the need for asymptotic expansions. It should be stated that this method does not make any a priori assumption on the structure of the solution; on the contrary, a multi-scale structure is the standard hypothesis in all classical techniques.

Recently, this method has made it possible to deal with different mathematical problems which were out of reach using traditional techniques (see [2], [3], [12], and [25]), for example in the field of fluid-structure interactions (see [2]), and of the asymptotic behavior of the spectrum of some elliptic operators with rapidly oscillating coefficients, where it has furnished a new understanding of the spectrum of the so-called composite materials (see [3]).

The method has also been used by F. Santosa and W. W. Symes (see [25]) in their study of dispersive effective media for wave propagation in periodic composites (see also [22], and [24]). In the framework of hyperbolic problems, this method gave rise to a neat quantitative analysis of dispersive effects which occur in large time scales, and this is now known as the dispersive effective medium theory.

In the specific field of periodic homogenization of elliptic operators in bounded domains without holes, this method has shed new light and offered an alternative way to view the classical approaches (see [10], and [11]). Moreover, the method allows one to go further, giving a rigorous mathematical justification of phenomena which are not adequately dealt with by the classical theory of homogenization (see [2], [3], and [12]).

This work is the first to use the Bloch wave method in the understanding of periodically perforated domains, and should allow further progress to be made in other open questions concerning the homogenization of perforated domains. Indeed, the machinery developed in this paper to understand classical results in the homogenization of perforated media should prove useful in other contexts, as it has in the fields cited above. 
To our knowledge, the homogenization problem considered in this paper was first studied in [8] for a two dimensional domain. The so-called energy method, introduced in [17], is used in the above mentioned reference to prove convergence. Other mathematical methods have been used to approach this problem: for example, the pioneering works of E. De Giorgi and S. Spagnolo concerning the classical notion of G-convergence for elliptic partial differential operators (see [14], and [26]) and more recently, the techniques of O. A. Oleinik et al. (see [19] Section II.2), and the concept of $\Gamma$-convergence which applies to energy functionals (see, e.g., [5] Section I.3, and [13]).

Proofs in these cited papers are mainly based on the existence of a uniformly bounded family of extension operators $P^{\varepsilon}, P^{\varepsilon}: H^{1}\left(\Omega^{\varepsilon}\right) \longrightarrow H^{1}(\Omega)$, and on the construction of some suitable test functions, in order to pass to the limit in the product of sequences that converge weakly in $L^{2}(\Omega)$ (see [4] to avoid the construction of $P^{\varepsilon}$ ). Nevertheless, behind all the above mentioned techniques are the classical two-scale asymptotic expansions (see A. Bensoussan, J. L. Lions, and G. Papanicolaou [6] and E. Sánchez-Palencia [23]).

By using Bloch wave decomposition, we completely avoid asymptotic expansions since the homogenized problem is obtained directly from the Hessian of the first Bloch eigenvalue. Bloch wave decomposition is used in [11] to study homogenization problems in bounded heterogeneous media without holes. We follow the main ideas introduced in [11]. Therefore, we first prove convergence for the case of the whole space " $\mathbb{R}^{N}$ minus the holes". The general case (where $\Omega$ is a bounded domain) is justified using a standard localization technique. It is important to mention that the methods of [11] cannot be applied directly: since we are dealing with a perforated domain, we are forced to develop an alternative approach.

The structure of our paper is as follows. In Section 2, we pose the homogenization problem and introduce the notations that we use throughout the paper. For the sake of simplicity, we consider a sequence of periodically perforated domains $\Omega^{\varepsilon}$, all of which have a fixed smooth external boundary that does not intersect the boundaries of the perforations (see Remark 3 in Section 4 for more general geometries). Some previous results obtained in [8] are introduced in Section 2 to verify that our results agree with those obtained by other methods. A new characterization of the homogenized matrix is obtained (see Proposition 2, Section 3) which coincides with the Hessian of the dominant Bloch eigenvalue at the origin, which is shown to be a critical point on the period representing the Bloch parameter.

In Section 3, we introduce the result of Bloch wave decomposition for the case of domains with holes (see Theorems 1 and 2). We also prove that all the information on the homogenized problem is contained in the first Bloch eigenvalue (see Proposition 1). The convergence of the solutions requires the first eigenvalue to be smooth with respect to the Bloch parameter $\eta$. This is proved using spectral perturbation theory. More precisely, it is proved in a 
neighborhood of the origin, that the first Bloch eigenvalue and its corresponding Bloch eigenfunction define analytic functions with respect to $\eta$ (see Theorem 3 ). Theorem 3 also provides an alternative way to prove this technical result of smoothness for the first Bloch eigenvalue appearing in [11]. See [27] for other results on the smoothness of certain Bloch wave families with respect to the Bloch parameter.

Finally, in Section 4, we find the homogenized problem and prove convergence of the solutions. However, it should be kept in mind that the total periodicity of the structure is essential in order to apply the Bloch wave decomposition technique; see [9] and [20] for different approaches which apply in the case of very small holes compared with the period of the structure.

2. Setting of the problem. Let $Y$ be the unit cell in $\mathbb{R}^{N}, Y=[0,2 \pi)^{N}$, and $T$, "the hole", an open bounded domain with a smooth boundary, $\partial T ; \bar{T} \subset Y$ and $d(\bar{T}, \partial Y)>0$. We denote by $Y^{*}$ the domain $Y^{*}=Y-\bar{T}$ and by $\theta$ the volume fraction material constant, $\theta=\left|Y^{*}\right| /|Y|$.

Let $Y_{\mathrm{k}}$ denote the translation of $Y$ to the point $\tilde{x}_{\mathrm{k}} \in 2 \pi \mathbb{Z}^{N}$ (the set of the points of $\mathbb{R}^{N}$ such that every coordinate is of the form $2 \pi n, n \in \mathbb{Z}$ ) and $T_{\mathrm{k}}$ denote the translation of $T$ to the point $\tilde{x}_{\mathrm{k}}, \bar{T}_{\mathrm{k}} \subset Y_{\mathrm{k}}$ and $Y_{\mathrm{k}}^{*}=Y_{\mathrm{k}}-\bar{T}_{\mathrm{k}}$. Let $Y_{\mathrm{k}}^{\varepsilon}$ $\left(T_{\mathrm{k}}^{\varepsilon}, Y_{\mathrm{k}}^{\varepsilon *}\right)$ be the homothetics of $Y_{\mathrm{k}}\left(T_{\mathrm{k}}, Y_{\mathrm{k}}^{*}\right), \varepsilon Y_{\mathrm{k}}\left(\varepsilon T_{\mathrm{k}}, \varepsilon Y_{\mathrm{k}}^{*}\right)$. In this way, $\mathbb{R}^{N}$ is covered periodically by cells $Y_{\mathrm{k}}^{\varepsilon}$, $\varepsilon$ being a small parameter that converges to 0 .

Let $\left\{a_{i j}\right\}_{i, j=1, \ldots, N}$ be $Y$-periodic bounded measurable real functions defined on $\mathbb{R}^{N}$ minus the holes, $a_{i j} \in L_{\#}^{\infty}\left(Y^{*}\right)$ satisfying the symmetry and ellipticity conditions

$$
\begin{gathered}
a_{i j}=a_{j i} \quad \text { for all } i, j=1, \ldots, N \text { and } \\
a_{i j}(y) \xi_{i} \xi_{j} \geq \alpha|\xi|^{2} \quad \text { for some } \alpha>0, \text { for all } \xi \in \mathbb{R}^{N} .
\end{gathered}
$$

Here, and henceforth, the assumption of the summation over repeated indices is performed.

Let $\Omega$ be an open bounded domain of $\mathbb{R}^{N}$ with a Lipschitz boundary. For the sake of simplicity, we assume that this boundary does not cut any hole $T_{\mathrm{k}}^{\varepsilon}$ (see Remark 3 in Section 4 for more general geometries). Let $N(\varepsilon)$ be the number of cells $Y_{\mathrm{k}}^{\varepsilon}$ contained in $\Omega ; N(\varepsilon)=O\left(\varepsilon^{-N}\right)$.

Let $\Omega^{\varepsilon}$ be $\Omega-\left(\bigcup_{\mathrm{k}} \bar{T}_{\mathrm{k}}^{\varepsilon} \cap \Omega\right)=\Omega-\bigcup_{\mathrm{k}=1}^{N(\varepsilon)} \bar{T}_{\mathrm{k}}^{\varepsilon}$ and $f \in L^{2}(\Omega)$. Let us denote by $a_{i j}^{\varepsilon}(x)$ the value of the coefficient $a_{i j}(y)$ at the point $x / \varepsilon$.

We consider the homogenization problem

$$
\begin{aligned}
& -\frac{\partial}{\partial x_{j}}\left(a_{i j}^{\varepsilon}(x) \frac{\partial u^{\varepsilon}}{\partial x_{i}}\right)=f \text { in } \Omega^{\varepsilon}, \\
& a_{i j}^{\varepsilon} \frac{\partial u^{\varepsilon}}{\partial x_{i}} n_{j}=0 \text { on } \partial T_{\mathrm{k}}^{\varepsilon}, \mathrm{k}=1,2, \ldots, N(\varepsilon), \\
& u^{\varepsilon}=0 \text { on } \partial \Omega,
\end{aligned}
$$


where $n$ denotes the outward unit normal to the boundary of the hole $T_{\mathrm{k}}^{\varepsilon}$.

Problem (2.1) has the equivalent variational formulation: Find $u^{\varepsilon} \in V^{\varepsilon}$, such that

$$
\int_{\Omega^{\varepsilon}} a_{i j}^{\varepsilon}(x) \frac{\partial u^{\varepsilon}}{\partial x_{i}} \frac{\partial \bar{v}}{\partial x_{j}} d x=\int_{\Omega^{\varepsilon}} f \bar{v} d x, \quad \text { for all } v \in V^{\varepsilon}
$$

$V^{\varepsilon}$ being the space $V^{\varepsilon}=\left\{u \in H^{1}\left(\Omega^{\varepsilon}\right) \mid u=0\right.$ on $\left.\partial \Omega\right\}$.

We study the asymptotic behavior of the solution of (2.1) by using the Bloch wave transform.

As pointed out in Section 1, the asymptotic behavior of the solution of (2.1) has already been studied in [8] by using the energy method and techniques introduced in [17] (see [4], [5], or [19] for other techniques). Other boundary conditions are considered for the same operator in [8], [18], and [19].

Here, we simply outline the results in [8]. All the results were obtained on the basis that there is an extension operator $P^{\varepsilon}, P^{\varepsilon}: V^{\varepsilon} \rightarrow H_{0}^{1}(\Omega)$ such that

$$
\left\|\nabla P^{\varepsilon} v\right\|_{L^{2}(\Omega)} \leq C\|\nabla v\|_{L^{2}\left(\Omega^{\varepsilon}\right)}, \quad \text { for all } v \in V^{\varepsilon} .
$$

Here, and henceforth, $C$ denotes different constants independent of $\varepsilon$.

On account of (2.2) and (2.3), $\left\|\nabla P^{\varepsilon} u^{\varepsilon}\right\|_{L^{2}(\Omega)} \leq C$. Hence, it is possible to extract a subsequence, still denoted by $\varepsilon, P^{\varepsilon} u^{\varepsilon}$ converging weakly in $H_{0}^{1}(\Omega)$, as $\varepsilon \rightarrow 0$, towards some function $u^{0}$. Then, it is proved that $u^{0}$ is the solution of the homogenized problem:

$$
\mid \begin{aligned}
& -a_{i j}^{h} \frac{\partial^{2} u^{0}}{\partial x_{i} \partial x_{j}}=f \theta \text { in } \Omega \\
& u^{0}=0 \text { on } \partial \Omega .
\end{aligned}
$$

The homogenized coefficients $a_{i j}^{h}$ in (2.4) satisfy the symmetry and ellipticity conditions and are defined by

$$
a_{k \ell}^{h}=\frac{1}{|Y|} \int_{Y^{*}} a_{i j}(y) \frac{\partial w^{k}}{\partial y_{i}} \frac{\partial w^{\ell}}{\partial y_{j}} d y, \quad k, \ell=1,2, \ldots, N,
$$

where $y$ is the so-called local variable, $y=x / \varepsilon$, and for each $k=1,2, \ldots, N$, $w^{k}$ is the solution of the local problem on the unit cell $Y$ :

$$
\begin{aligned}
& -\frac{\partial}{\partial y_{j}}\left(a_{i j}(y) \frac{\partial w^{k}}{\partial y_{i}}\right)=0 \text { in } Y^{*} \\
& a_{i j} \frac{\partial w^{k}}{\partial y_{j}} n_{i}=0 \text { on } \partial T \\
& w^{k}-e^{k} \cdot y \text { is } Y \text {-periodic. }
\end{aligned}
$$


Let us consider $v^{k}=w^{k}-e^{k} \cdot y, e^{k}$ being the unit vector in the $k$-th direction, $e_{i}^{k}=\delta_{i k} . v^{k}$ is the unique solution (defined up to an additive constant) of the problem:

$$
\int_{Y^{*}} a_{i j} \frac{\partial v^{k}}{\partial y_{i}} \frac{\partial \bar{v}}{\partial y_{j}} d y=-\int_{Y^{*}} a_{j k} \frac{\partial \bar{v}}{\partial y_{j}} d y, \quad \text { for all } v \in H_{\#}^{1}\left(Y^{*}\right) .
$$

We denote by $H_{\#}^{1}\left(Y^{*}\right)$ the space

$$
H_{\#}^{1}\left(Y^{*}\right)=\left\{u \in H_{\mathrm{loc}}^{1}\left(\mathbb{R}^{N}-\bigcup_{\mathrm{k}} \bar{T}_{\mathrm{k}}\right) \mid u \text { is } Y \text {-periodic }\right\},
$$

where the union is extended to all the holes $T_{\mathrm{k}}$ in $\mathbb{R}^{N}$. In the same way,

$$
L_{\#}^{2}\left(Y^{*}\right)=\left\{u \in L_{\text {loc }}^{2}\left(\mathbb{R}^{N}-\bigcup_{\mathrm{k}} \bar{T}_{\mathrm{k}}\right) \mid u \text { is } Y \text {-periodic }\right\} .
$$

The norms in $H_{\#}^{1}\left(Y^{*}\right)$ and $L_{\#}^{2}\left(Y^{*}\right)$ are those of $H^{1}\left(Y^{*}\right)$ and $L^{2}\left(Y^{*}\right)$ respectively.

From (2.5) and (2.7) it is easy to prove the formula which we shall use in Sections 3 and 4 ,

$$
a_{k \ell}^{h}=\frac{1}{|Y|} \int_{Y^{*}} a_{k j}(y) \frac{\partial w^{\ell}}{\partial y_{j}} d y, \quad k, \ell=1,2, \ldots, N,
$$

for the homogenized coefficients.

As noted in [8], on account of the uniqueness of the solution of (2.4) in $H_{0}^{1}(\Omega)$, the convergence, as $\varepsilon \rightarrow 0$, of the whole sequence $P^{\varepsilon} u^{\varepsilon}$ towards $u^{0}$ in $H_{0}^{1}(\Omega)$ weak holds and this limit does not depend on the extension operator satisfying (2.3).

The Bloch transform allows us to obtain the same result avoiding both the use of the test functions $w^{k}$, and the problem of passing to the limit in the product of two sequences which converge weakly in $L^{2}(\Omega)$. In fact, in Section 4 we prove that, if we extend $u^{\varepsilon}$ by zero in $\bigcup_{\mathrm{k}} T_{\mathrm{k}}^{\varepsilon}$, the weak limit of this sequence (that we shall denote by $\tilde{u}^{\varepsilon}$ ) in $L^{2}(\Omega)$ is the solution of $(2.4)$.

As the local problem (2.6) is obtained from the microstructure of the problem and allows us to obtain macroscopic information, we need to introduce new problems giving us microscopic information. We get this information from the so-called Bloch eigenvector and Bloch eigenvalue: the homogenized coefficients and the solution of the local problem are given through the first Bloch eigenvalue and eigenvector respectively (see Remark 1 and Proposition 2 in Section 3).

3. The Bloch transform and the first Bloch eigenvalue. Let $a_{i j}, Y$, $T$, and $Y^{*}$ be as in Section 2. For simplicity, we denote by $\mathbb{R}^{N}-\bigcup_{\mathrm{k}} \bar{T}_{\mathrm{k}}$ the space 
$\mathbb{R}^{N}$ minus all the holes $\bar{T}_{\mathrm{k}} \subset Y_{\mathrm{k}}, Y_{\mathrm{k}}$ being the translated cell of $Y$ to the point $\tilde{x}_{\mathrm{k}}, \tilde{x}_{\mathrm{k}} \in 2 \pi \mathbb{Z}^{N}$.

Let $A$ be the operator

$$
A=-\frac{\partial}{\partial y_{j}}\left(a_{i j}(y) \frac{\partial}{\partial y_{i}}\right) \quad \text { in }\left(\mathbb{R}^{N}-\bigcup_{\mathrm{k}} \bar{T}_{\mathrm{k}}\right)
$$

with a homogeneous Neumann condition on the boundary of the holes, i.e.,

$$
\left(a_{i j}(y) \frac{\partial}{\partial y_{i}}\right) n_{j}=0 \quad \text { on } \partial T_{\mathrm{k}}, \text { for all } \mathrm{k}
$$

More specifically, $A+I$ denotes the Lax-Milgram operator associated with the sesquilinear, hermitian, continuous, and coercive form on $V$

$$
b(u, v)=\int_{\left(\mathbb{R}^{N}-\bigcup_{\mathrm{k}} \bar{T}_{\mathrm{k}}\right)}\left(a_{i j} \frac{\partial u}{\partial y_{i}} \frac{\partial \bar{v}}{\partial y_{j}}+u \bar{v}\right) d y,
$$

where $I$ is the identity operator on $H$, and

$$
V=H^{1}\left(\mathbb{R}^{N}-\bigcup_{\mathrm{k}} \bar{T}_{\mathrm{k}}\right), \quad H=L^{2}\left(\mathbb{R}^{N}-\bigcup_{\mathrm{k}} \bar{T}_{\mathrm{k}}\right) .
$$

For each $\eta, \eta \in Y^{\prime}, Y^{\prime}$ being the dual cell to $Y, Y^{\prime}=\left[-\frac{1}{2}, \frac{1}{2}\right)^{N}$, we consider $A(\eta)$ the shifted operator,

$$
A(\eta)=-\left(\frac{\partial}{\partial y_{i}}+\mathbf{i} \eta_{i}\right)\left[a_{i j}(y)\left(\frac{\partial}{\partial y_{j}}+\mathbf{i} \eta_{j}\right)\right] \quad \text { in }\left(\mathbb{R}^{N}-\bigcup_{\mathrm{k}} \bar{T}_{\mathrm{k}}\right),
$$

acting on the $Y$-periodic functions, with the boundary conditions

$$
a_{i j}\left(\frac{\partial}{\partial y_{i}}+\mathbf{i} \eta_{i}\right) n_{j}=0 \quad \text { on } \partial T_{\mathrm{k}}, \text { for all } \mathrm{k} .
$$

In fact, $A(\eta)$ is the operator associated with the sesquilinear, continuous, hermitian, and positive form $a(\eta ; \cdot, \cdot)$ on $H_{\#}^{1}\left(Y^{*}\right)$,

$$
a(\eta ; u, v)=\int_{Y^{*}} a_{i j}(y)\left(\frac{\partial u}{\partial y_{i}}+\mathbf{i} \eta_{i} u\right)\left(\frac{\partial \bar{v}}{\partial y_{j}}-\mathbf{i} \eta_{j} \bar{v}\right) d y .
$$

Taking into account that the embedding $H_{\#}^{1}\left(Y^{*}\right) \subset L_{\#}^{2}\left(Y^{*}\right)$ is dense and compact, and that $a(\eta ; \cdot, \cdot)+\mu(\cdot, \cdot)_{L_{\#}^{2}\left(Y^{*}\right)}$ is coercive for large enough $\mu$, the operator $A(\eta)$ is a self-adjoint operator on $L_{\#}^{2}\left(Y^{*}\right)$ with a compact resolvent, and it has 
a discrete spectrum, $\left\{\lambda_{m}(\eta)\right\}_{m=1}^{\infty}$; the corresponding eigenfunctions are a basis for both spaces $L_{\#}^{2}\left(Y^{*}\right)$ and $H_{\#}^{1}\left(Y^{*}\right)$.

Let $0 \leq \lambda_{1}(\eta) \leq \lambda_{2}(\eta) \leq \cdots \leq \lambda_{m}(\eta) \leq \cdots \rightarrow \infty$ be the sequence of eigenvalues of the problem

$$
\begin{aligned}
& A(\eta) \phi=\lambda \phi \text { in }\left(\mathbb{R}^{N}-\bigcup_{\mathrm{k}} \bar{T}_{\mathrm{k}}\right), \\
& \phi \text { is } Y \text {-periodic, }
\end{aligned}
$$

with the classical convention of repeated eigenvalues. Let $\left\{\phi_{m}(\cdot, \eta)\right\}_{m=1}^{\infty}$ denote the corresponding eigenfunctions that form an orthonormal basis in $L_{\#}^{2}\left(Y^{*}\right)$.

Remark 1. As usual, $\left\{\lambda_{m}(\eta)\right\}_{m \geq 1}$ are referred to as the Bloch eigenvalues and $\left\{\phi_{m}(\cdot, \eta)\right\}_{m=1}^{\infty}$ as the Bloch eigenvectors or the Bloch waves; Bloch parameter $\eta$ varies in the dual cell $Y^{\prime}=\left[-\frac{1}{2}, \frac{1}{2}\right)^{N}$. The functions $e^{\mathbf{i} \eta \cdot y} \phi_{m}(y, \eta)$ are the socalled generalized eigenfunctions of $A$ associated with the generalized eigenvalues $\lambda_{m}(\eta)$ :

$$
A\left(e^{\mathbf{i} \eta \cdot y} \phi_{m}(y, \eta)\right)=e^{\mathbf{i} \eta \cdot y} \lambda_{m}(\eta) \phi_{m}(y, \eta)
$$

In addition, we observe that problem (3.2) is equivalent to the spectral problem: Find $\lambda=\lambda(\eta) \in \mathbb{R}^{N}$ and $\psi=\psi(y, \eta)$ ( $\psi$ not identically zero) such that

$$
\begin{aligned}
& A \psi(\cdot, \eta)=\lambda \psi(\cdot, \eta) \text { in }\left(\mathbb{R}^{N}-\bigcup_{\mathrm{k}} \bar{T}_{\mathrm{k}}\right), \\
& \psi(\cdot, \eta) \text { is }(\eta, Y) \text {-periodic, i.e., for all } m \in \mathbb{Z}^{N}, y \in \mathbb{R}^{N}, \\
& \psi(y+2 \pi m, \eta)=e^{2 \pi \mathrm{i} m \cdot \eta} \psi(y, \eta) .
\end{aligned}
$$

The eigenfunctions of (3.4) associated with the eigenvalues $\lambda_{m}(\eta)$ are $\psi_{m}(y, \eta)=$ $e^{i \eta \cdot y} \phi_{m}(y, \eta)$. The Neumann condition on the boundary of the holes for the solution of (3.4),

$$
a_{i j} \frac{\partial \psi_{m}}{\partial y_{j}} n_{i}=0 \quad \text { on } \partial T
$$

reads

$$
a_{i j}\left(\frac{\partial \phi_{m}}{\partial y_{j}}+\mathbf{i} \eta_{j} \phi_{m}\right) n_{i}=0 \quad \text { on } \partial T \text {. }
$$

In the following theorem, we use the above parameterized eigenvalues of (3.2) $\lambda_{m}(\eta)$ to describe the spectral resolution of $A$ as an unbounded self-adjoint operator on $L^{2}\left(\mathbb{R}^{N}-\bigcup_{\mathrm{k}} \bar{T}_{\mathrm{k}}\right)$. 
Theorem 1. Let $g \in L^{2}\left(\mathbb{R}^{N}-\bigcup_{\mathrm{k}} \bar{T}_{\mathrm{k}}\right)$. The function $g$ admits the expansion in Bloch waves:

$$
g(y)=\sum_{m=1}^{\infty} \int_{Y^{\prime}} \hat{g}_{m}(\eta) e^{\mathbf{i} \eta \cdot y} \phi_{m}(y, \eta) d \eta
$$

where the $m$-th Bloch coefficient is given by

$$
\hat{g}_{m}(\eta)=\int_{\left(\mathbb{R}^{N}-\bigcup_{\mathrm{k}} \bar{T}_{\mathrm{k}}\right)} g(y) e^{-\mathbf{i} \eta \cdot y} \bar{\phi}_{m}(y, \eta) d y .
$$

Moreover, the following Parseval Identity holds:

$$
\int_{\left(\mathbb{R}^{N}-\cup_{\mathrm{k}} \bar{T}_{\mathrm{k}}\right)}|g(y)|^{2} d y=\int_{Y^{\prime}} \sum_{m=1}^{\infty}\left|\hat{g}_{m}(\eta)\right|^{2} d \eta .
$$

In addition, for each $g$ belonging to the domain of operator $A$

$$
A g(y)=\sum_{m=1}^{\infty} \int_{Y^{\prime}} \lambda_{m}(\eta) \hat{g}_{m}(\eta) e^{\mathrm{i} \eta \cdot y} \phi_{m}(y, \eta) d \eta \quad \text { in } L^{2}\left(\mathbb{R}^{N}-\bigcup_{\mathrm{k}} \bar{T}_{\mathrm{k}}\right)
$$

Proof. As pointed out in [21] Section IV.5, the proof of formulas (3.5)-(3.8) holds as in the classical Bloch expansion with minor modifications (see, e.g., Section IV.3 in [6] and Section IV.5 in [21]).

We note that the expansions (3.5)-(3.8) are written in terms of the "microscopic variable" $y$. The following theorem gives us these expansions in terms of the "macroscopic variable" $x, x=\varepsilon y$. In order to state this result it is useful to introduce here the notations:

$$
y=\frac{x}{\varepsilon}, \quad \xi=\frac{\eta}{\varepsilon}
$$

$$
\lambda_{m}^{\varepsilon}(\xi)=\frac{1}{\varepsilon^{2}} \lambda_{m}(\eta), \quad \phi_{m}^{\varepsilon}(x, \xi)=\phi_{m}(y, \eta),
$$

where $y \in\left(\mathbb{R}^{N}-\bigcup_{\mathrm{k}} \bar{T}_{\mathrm{k}}\right), x \in\left(\mathbb{R}^{N}-\bigcup_{\mathrm{k}} \bar{T}_{\mathrm{k}}^{\varepsilon}\right)\left(y \in Y^{*} \Leftrightarrow x \in \varepsilon Y^{*}\right), \eta \in Y^{\prime}$, $\xi \in Y^{\prime} / \varepsilon$.

Let $A^{\varepsilon}$ denote the operator $\left(1 / \varepsilon^{2}\right) A$ in the $x$ variable:

$$
A^{\varepsilon} g(x)=\frac{1}{\varepsilon^{2}} A(g(\varepsilon y)) \text {. }
$$

That is to say,

$$
A^{\varepsilon}=-\frac{\partial}{\partial x_{i}}\left(a_{i j}^{\varepsilon}(x) \frac{\partial}{\partial x_{j}}\right) \quad \text { in }\left(\mathbb{R}^{N}-\bigcup_{\mathrm{k}} \bar{T}_{\mathrm{k}}^{\varepsilon}\right),
$$


with the boundary conditions on the holes $T_{\mathrm{k}}^{\varepsilon}$ :

$$
a_{i j}^{\varepsilon} \frac{\partial}{\partial x_{j}} n_{i}=0 \quad \text { on } \partial T_{\mathrm{k}}^{\varepsilon}, \text { for all k. }
$$

By introducing the change of variable (3.9) into (3.5)-(3.8) and taking into account (3.10), the statements in the following theorem hold.

Theorem 2. Let $g \in L^{2}\left(\mathbb{R}^{N}-\bigcup_{\mathrm{k}} \bar{T}_{\mathrm{k}}^{\varepsilon}\right)$. Then $g$ admits the expansion:

$$
g(x)=\varepsilon^{N / 2} \sum_{m=1}^{\infty} \int_{Y^{\prime} / \varepsilon} \hat{g}_{m}^{\varepsilon}(\xi) e^{\mathbf{i} x \cdot \xi} \phi_{m}^{\varepsilon}(x, \xi) d \xi,
$$

where $\hat{g}_{m}^{\varepsilon}(\xi)$ denotes the $m$-th Bloch coefficient

$$
\hat{g}_{m}^{\varepsilon}(\xi)=\varepsilon^{-N / 2} \int_{\left(\mathbb{R}^{N}-\cup_{\mathrm{k}} \bar{T}_{\mathrm{k}}^{\varepsilon}\right)} g(x) e^{-\mathbf{i} x \cdot \xi} \bar{\phi}_{m}^{\varepsilon}(x, \xi) d x .
$$

Moreover, the Plancherel identity holds

$$
\varepsilon^{-N} \int_{\left(\mathbb{R}^{N}-\cup_{\mathrm{k}} \bar{T}_{\mathrm{k}}^{\varepsilon}\right)} g(x) \overline{h(x)} d x=\int_{Y^{\prime} / \varepsilon} \sum_{m=1}^{\infty} \hat{g}_{m}^{\varepsilon}(\xi) \overline{\hat{h}_{m}^{\varepsilon}(\xi)} d \xi
$$

for all $g, \in L^{2}\left(\mathbb{R}^{N}-\bigcup_{\mathrm{k}} \bar{T}_{\mathrm{k}}^{\varepsilon}\right)$. In addition, we have

$$
A^{\varepsilon} u(x)=\varepsilon^{N / 2} \sum_{m=1}^{\infty} \int_{Y^{\prime} / \varepsilon} \lambda_{m}^{\varepsilon}(\xi) \hat{u}_{m}^{\varepsilon}(\xi) e^{\mathbf{i} x \cdot \xi} \phi_{m}^{\varepsilon}(x, \xi) d \xi,
$$

for each $u \in\left\{v \in L^{2}\left(\mathbb{R}^{N}-\bigcup_{\mathrm{k}} \bar{T}_{\mathrm{k}}^{\varepsilon}\right) \mid A^{\varepsilon} v \in L^{2}\left(\mathbb{R}^{N}-\bigcup_{\mathrm{k}} \bar{T}_{\mathrm{k}}^{\varepsilon}\right)\right\}$.

The following proposition leads us to assert that the homogenized problem can be obtained through the limit behavior, as $\varepsilon \rightarrow 0$, of the first Bloch eigenvalue and its corresponding Bloch eigenfunction in formulas (3.11), (3.12), and (3.14). This result was already envisaged in [25] for a hyperbolic problem in an unbounded domain.

Proposition 1. Let $f$ be a function of $L^{2}\left(\mathbb{R}^{N}\right)$ and let $u^{\varepsilon}$ be the solution of $A^{\varepsilon} u^{\varepsilon}=f$ in $\left(\mathbb{R}^{N}-\bigcup_{\mathrm{k}} \bar{T}_{\mathrm{k}}^{\varepsilon}\right)$, that is to say, the solution of

$$
\begin{aligned}
& -\frac{\partial}{\partial x_{j}}\left(a_{i j}^{\varepsilon}(x) \frac{\partial u^{\varepsilon}}{\partial x_{i}}\right)=f \text { in }\left(\mathbb{R}^{N}-\bigcup_{\mathrm{k}} \bar{T}_{\mathrm{k}}^{\varepsilon}\right), \\
& a_{i j}^{\varepsilon} \frac{\partial u^{\varepsilon}}{\partial x_{i}} n_{j}=0 \text { on } \partial T_{\mathrm{k}}^{\varepsilon}, \text { for all } \mathrm{k},
\end{aligned}
$$


$u^{\varepsilon} \in H^{1}\left(\mathbb{R}^{N}-\bigcup_{\mathrm{k}} \bar{T}_{\mathrm{k}}^{\varepsilon}\right)$. Then,

$$
\left\|\varepsilon^{N / 2} \sum_{m=2}^{\infty} \int_{Y^{\prime} / \varepsilon} \hat{u}_{m}^{\varepsilon} e^{\mathbf{i} x \cdot \xi} \phi_{m}^{\varepsilon}(x, \xi) d \xi\right\|_{L^{2}\left(\mathbb{R}^{N}-\bigcup_{\mathbf{k}} \bar{T}_{\mathrm{k}}^{\varepsilon}\right)} \leq C_{1} \varepsilon^{2},
$$

where $C_{1}$ is a constant that does not depend on $\varepsilon$.

Proof. On account of $A^{\varepsilon} u^{\varepsilon}=f$ and Theorem 2, it follows that

$$
\lambda_{m}^{\varepsilon}(\xi) \hat{u}_{m}^{\varepsilon}(\xi)=\hat{f}_{m}^{\varepsilon}(\xi),
$$

thus,

$$
\varepsilon^{N} \int_{Y^{\prime} / \varepsilon} \sum_{m=2}^{\infty}\left|\hat{u}_{m}^{\varepsilon}(\xi)\right|^{2} d \xi=\varepsilon^{N} \int_{Y^{\prime} / \varepsilon} \sum_{m=2}^{\infty}\left|\frac{\hat{f}_{m}^{\varepsilon}(\xi)}{\lambda_{m}^{\varepsilon}(\xi)}\right|^{2} d \xi .
$$

Now, the minimax principle leads us to obtain:

$$
\lambda_{m}(\eta) \geq \lambda_{2}(\eta) \geq \lambda_{2}^{*} \text { for all } m \geq 2, \eta \in Y^{\prime} .
$$

Taking into account (3.10), (3.13), and (3.17), we have

$$
\varepsilon^{N} \int_{Y^{\prime} / \varepsilon} \sum_{m=2}^{\infty}\left|\hat{u}_{m}^{\varepsilon}(\xi)\right|^{2} d \xi \leq C \varepsilon^{4},
$$

that is, (3.16) in the statement of the proposition holds.

In order to prove (3.17) we consider the set of subspace of dimension 2 of $H^{1}(Y): \tilde{E}_{2}=\left[u_{1} e^{\mathrm{i} \eta \cdot y}, u_{2} e^{\mathrm{i} \cdot \cdot y}\right]$, where $u_{1}, u_{2}$ are two linearly independent functions in $H_{\#}^{1}(Y), E_{2}=\left[u_{1}, u_{2}\right]$. Let $\lambda_{2}^{*}$ be the second eigenvalue of the Neumann problem on $Y^{*}$ :

$$
\begin{aligned}
& -\frac{\partial}{\partial y_{j}}\left(a_{i j}(y) \frac{\partial u}{\partial y_{i}}\right)=\lambda u \text { in } \stackrel{\circ}{Y^{*}} \\
& a_{i j} \frac{\partial u}{\partial y_{i}} n_{j}=0 \text { on } \partial Y^{*} .
\end{aligned}
$$

Obviously,

$$
\begin{aligned}
& \lambda_{2}^{*} \leq \min _{\tilde{E}_{2} \subset H^{1}(Y)} \max _{\substack{u \in \tilde{E}_{2} \\
u \neq 0}} \frac{\int_{Y^{*}} a_{i j}(y) \frac{\partial u}{\partial y_{i}} \frac{\partial \bar{u}}{\partial y_{j}} d y}{\int_{Y^{*}}|u|^{2} d y} \\
& =\min _{\substack{E_{2} \subset H_{\#}^{1}(Y) \\
\operatorname{dim} E_{2}=2}} \max _{\substack{u \in E_{2} \\
u \neq 0}} \frac{a(\eta ; u, u)}{\int_{Y^{*}}|u|^{2} d y}=\lambda_{2}(\eta) .
\end{aligned}
$$

Here $a(\eta ; \cdot, \cdot)$ is the form defined in (3.1). Therefore, (3.17) holds as well as the result of Proposition 1. 
It will prove useful in Section 4, to introduce here the following results for the smoothness of the first Bloch eigenvalue with respect to the Bloch parameter $\eta$ (see Theorem 3) and its connection with the local problem (2.6) (see Proposition 2 ). Proposition 3 deals with the relation between the first Bloch coefficient and the Fourier transform.

Let us first notice that for $\eta=0$, the first eigenvalue of problem (3.2) is $\lambda_{1}(0)=0$ and the corresponding eigenfunctions are the constants. We have chosen $\phi_{1}(\cdot, 0)$ as the normalized function in $L_{\#}^{2}(Y)$,

$$
\phi_{1}(\cdot, 0)=\frac{1}{|Y|^{1 / 2}}=\frac{1}{(2 \pi)^{N / 2}} \text {. }
$$

Thus, we are led to choose the normalization of the Fourier transform:

$$
\mathcal{F}[g](\xi)=\frac{1}{(2 \pi)^{N / 2}} \int_{\mathbb{R}^{N}} g(x) e^{-\mathbf{i} x \cdot \xi} d x,
$$

for all $g \in L^{2}\left(\mathbb{R}^{N}\right)$.

We use the technique in Section V.4 of [21] to prove the result in the next theorem. This technique is based on the reduction of the problem to an eigenvalue problem in a space of finite dimension. In order to make the exposition self-contained, we introduce here two lemmas that we shall use for the proof of Theorem 3. See Sections I.4 and VII.1 in [16], together with Section II.2 in [15], for the proof and for more general situations.

Lemma 1. Let $V$ be a Hilbert space and $z$ be a complex parameter, $z=$ $\left(z_{1}, z_{2}, \ldots, z_{N}\right), z \in \mathbb{C}^{N}$. Let $B(z)$ be a holomorphic function in a neighborhood of $z_{0}$ with values in $\mathcal{L}\left(V, V^{\prime}\right)$ and such that the inverse $B\left(z_{0}\right)^{-1} \in \mathcal{L}\left(V^{\prime}, V\right)$. Then, $B(z)^{-1}$ defines a function with values in $\mathcal{L}\left(V^{\prime}, V\right)$, depending holomorphically on $z$ for small enough $\left|z-z_{0}\right|$.

Lemma 2. Let $V$ be a Hilbert space and $P$ and $Q$ be two projections such that $\|P-Q\|_{\mathcal{L}(V)}<1$. The following assertions hold:

- The ranges $R(P)$ and $R(Q)$ have the same dimension.

- There is an invertible operator $U$ such that $U P U^{-1}=Q\left(U^{-1} Q U=P\right.$ respectively). In particular, $U\left(U^{-1}\right.$ respectively) maps $R(P)$ onto $R(Q)$ $(R(Q)$ onto $R(P)$ respectively $)$.

- If we consider $P=P(z), P(z)$ being a projection depending holomorphically on $z$, where $z$ is a complex parameter, $z \in \mathbb{C}^{N}$, then $U=U(z)$ also depends holomorphically on $z$. 
Theorem 3. There is a neighborhood $\mathcal{D}$ of the origin where the first eigenvalue $\lambda_{1}(\eta)$ of problem (3.2) remains simple and defines an analytic function of $\eta$. Besides, the first eigenvector $\phi_{1}(\cdot, \eta)$ can be chosen in such a way that the map

$$
\begin{aligned}
& \mathcal{D} \longrightarrow H_{\#}^{1}\left(Y^{*}\right) \\
& \eta \longrightarrow \phi_{1}(\cdot, \eta)
\end{aligned}
$$

is analytic and $\phi_{1}(\cdot, 0)=|Y|^{-1 / 2}$.

Proof. Let us consider the pair of spaces $H=L_{\#}^{2}\left(Y^{*}\right), V=H_{\#}^{1}\left(Y^{*}\right)$, and $A(\eta)$ the operator associated with the form $a(\eta ; \cdot, \cdot)$ in $(3.1)$. Obviously, there is a neighborhood of $\eta=0, \mathcal{U} \subset \mathbb{C}^{N}$, such that

$$
a(\eta ; u, v) \text { is holomorphic for any fixed } u, v \in V .
$$

Thanks to Hartogs Theorem for several complex variables $\left(\eta_{1}, \eta_{2}, \ldots, \eta_{N}\right)$ and the principle of uniform boundedness (see Section V.1 in [21] and Section II.2 in [15]), we can assert that the map $\eta \rightarrow A(\eta)$ defines a holomorphic function on $\mathcal{U}$ with values in $\mathcal{L}\left(V, V^{\prime}\right)$.

Let $\lambda_{0}=0$ be the first eigenvalue of the unperturbed operator, $A(0)$. We can construct a simple curve $\gamma$, in the plane of the spectral parameter $\xi$, enclosing only this eigenvalue of $A(0)$ and none other.

As the resolvent $(A(0)-\xi)^{-1} \in \mathcal{L}\left(V^{\prime}, V\right)$ is defined for $\xi \in \gamma$, we can prove that for small enough $|\eta|$ and $\xi$ in a sufficiently narrow annulus-shaped domain around $\gamma$, the resolvent $(A(\eta)-\xi)^{-1}$ defines a holomorphic function of $\eta$ and $\xi$ with values in $\mathcal{L}\left(V^{\prime}, V\right)$ (this result is a consequence of Hartogs Theorem and Lemma 1).

Let us consider the projection associated with the curve $\gamma, P_{\gamma}(\eta)$, defined by:

$$
P_{\gamma}(\eta)=-\frac{1}{2 \pi \mathbf{i}} \int_{\gamma}(A(\eta)-\xi)^{-1} d \xi
$$

$P(\eta)$ is a holomorphic function of $\eta$, for small $|\eta|$, with values in $\mathcal{L}(V)$.

Taking into account that $\lambda_{0}=0$ is a point within $\gamma, P(0) \neq 0$; therefore, for small enough $|\eta|, P(\eta) \neq 0$. Moreover, we can assert $\|P(\eta)-P(0)\|_{\mathcal{L}(V)}<1$, for $\eta$ in some neighborhood of the origin. We apply Lemma 2 to deduce that the ranges $R(P(0))$ and $R(P(\eta))$ have the same dimension 1; that is to say, on account of the properties of separation of the spectrum of $A(\eta)$ by a curve $\gamma$, there is only one eigenvalue $\lambda_{1}(\eta)$ of $A(\eta)$ inside $\gamma$. Let us consider $\mathcal{D}$ the smallest of all these neighborhoods of the origin for the $\eta$ variable.

Identifying $Q=P(0)$, Lemma 2 leads us to assert that the function of transformation $U(\eta)$ depends holomorphically on $\eta$, and solving

$$
A(\eta) \phi=\lambda_{1}(\eta) \phi
$$


with $\lambda_{1}(\eta)$ inside $\gamma$, is equivalent to solving the eigenvalue problem

$$
P(0) U(\eta) A(\eta) U(\eta)^{-1} P(0) u=\lambda u
$$

in the space $R(P(0))$. But, (3.25) is equivalent to looking for $\mu=\mu(\eta) \neq 0$ and $\lambda=\lambda(\eta)$ such that

$$
(\alpha(\eta)-\lambda) \mu=0
$$

where $\alpha(\eta)$ is some complex function depending holomorphically on $\eta$. The corresponding eigenfunction of (3.25) is $u(\eta)=\mu(\eta) \mathbf{e}$, with $\mathbf{e}$ an element of $R(P(0))$ and $\mu(\eta)$ some constant; and in (3.24),

$$
\phi(\cdot, \eta)=U(\eta)^{-1} u(\eta) .
$$

Therefore, in (3.26), (3.24), we have that $\lambda_{1}(\eta)=\lambda(\eta)=\alpha(\eta)$ depends holomorphically on $\eta$ and the eigenfunction $\phi(\cdot, \eta)$ can be chosen depending holomorphically on $\eta$ and such that $\phi(\cdot, 0)=1 /(2 \pi)^{N / 2}$. Hence the result of the theorem is true for some suitable neighborhood $\mathcal{D}$ of $\eta=0$.

Proposition 2. The first Bloch eigenvalue $\lambda_{1}(\eta)$ and eigenvector $\phi_{1}(\cdot, \eta)$ of problem (3.2) satisfy the following relations

$$
\begin{aligned}
\frac{\partial \lambda_{1}}{\partial \eta_{k}}(0) & =0, & k=1,2, \ldots, N, \\
\frac{1}{2} \frac{\partial^{2} \lambda_{1}}{\partial \eta_{k} \partial \eta_{\ell}}(0) & =a_{k \ell}^{h}, & k, \ell=1,2, \ldots, N, \\
\frac{\partial \phi_{1}}{\partial \eta_{k}}(\cdot, 0) & =\mathbf{i}|Y|^{-1 / 2} v^{k}, & k=1,2, \ldots, N,
\end{aligned}
$$

$a_{k \ell}^{h}$ being the homogenized coefficients defined in (2.5) and $v^{k}=w^{k}-e^{k} \cdot y$ the solution of (2.7).

Proof. On account of $A(\eta) \phi_{1}(\eta)=\lambda_{1}(\eta) \phi_{1}(\eta)$ and the variational formulation of (3.2):

$$
\int_{Y^{*}} a_{i j}\left(\frac{\partial \phi_{1}}{\partial y_{i}}+\mathbf{i} \eta_{i} \phi_{1}\right)\left(\frac{\partial \bar{\psi}}{\partial y_{j}}-\mathbf{i} \eta_{j} \bar{\psi}\right) d y=\lambda_{1}(\eta) \int_{Y^{*}} \phi_{1} \bar{\psi} d y
$$

for all $\psi \in H_{\#}^{1}\left(Y^{*}\right)$. We take derivatives with respect to $\eta_{k}$ in (3.31) and we obtain

$$
\begin{aligned}
\left(\frac{\partial \lambda_{1}}{\partial \eta_{k}} \phi_{1}+\frac{\partial \phi_{1}}{\partial \eta_{k}} \lambda_{1}, \psi\right)_{L^{2}\left(Y^{*}\right)}=a( & \left.\eta \frac{\partial \phi_{1}}{\partial \eta_{k}}, \psi\right)+2 \eta_{j} \int_{Y^{*}} a_{j k} \phi_{1} \bar{\psi} d y \\
& +\mathbf{i} \int_{Y^{*}}\left(a_{k j} \phi_{1} \frac{\partial \bar{\psi}}{\partial y_{j}}-a_{k i} \frac{\partial \phi_{1}}{\partial y_{i}} \bar{\psi}\right) d y
\end{aligned}
$$


Taking $\psi=\phi_{1}(\cdot, \eta)$ and making $\eta=0$, and since $\phi_{1}(\cdot, 0)$ is a constant, we obtain in (3.32) $\partial \lambda_{1} / \partial \eta_{k}(0)=0$, i.e. (3.28) is proved.

Since $\lambda_{1}(0)=0$ and (3.28), and making $\eta=0$ in (3.32), we have

$$
a\left(\frac{\partial \phi_{1}}{\partial \eta_{k}}(\cdot, 0), \psi\right)=-\frac{\mathbf{i}}{(2 \pi)^{N / 2}} \int_{Y^{*}} a_{k j} \frac{\partial \bar{\psi}}{\partial y_{j}} d y, \quad \text { for all } \psi \in H_{\#}^{1}\left(Y^{*}\right),
$$

so that, on account of $(2.7), \partial \phi_{1} / \partial \eta_{k}(\cdot, 0)=v^{k} \mathbf{i} /(2 \pi)^{N / 2}$ and (3.30) holds.

In order to prove $(3.29)$, we take derivatives with respect to $\eta_{\ell}$ in $(3.32)$ and make $\eta=0$ and $\psi=\phi_{1}(\cdot, 0)$; thus, taking into account (3.28), (3.30), we obtain

$$
\frac{\partial^{2} \lambda_{1}}{\partial \eta_{\ell} \partial \eta_{k}}(0)=\frac{1}{(2 \pi)^{N}}\left[\int_{Y^{*}} a_{j k} \frac{\partial w^{\ell}}{\partial y_{j}} d y+\int_{Y^{*}} a_{\ell j} \frac{\partial w^{k}}{\partial y_{j}} d y\right]
$$

and, on account of (2.8),

$$
\frac{\partial^{2} \lambda_{1}}{\partial \eta_{\ell} \partial \eta_{k}}(0)=2 a_{k \ell}^{h}
$$

Therefore, (3.29) in the statement of the proposition also holds.

Proposition 3. Let $\left\{g^{\varepsilon}\right\}_{\varepsilon}$ be a sequence $g^{\varepsilon} \in L^{2}\left(\mathbb{R}^{N}-\bigcup_{\mathrm{k}} \bar{T}_{\mathrm{k}}^{\varepsilon}\right)$. Let $\tilde{g}^{\varepsilon}$ be the extension by zero on $T_{\mathrm{k}}^{\varepsilon}$ of $g^{\varepsilon}$. We assume that $\tilde{g}^{\varepsilon}$ converges weakly in $L^{2}\left(\mathbb{R}^{N}\right)$ towards some function $g \in L^{2}\left(\mathbb{R}^{N}\right)$, as $\varepsilon \rightarrow 0$, and supp $g^{\varepsilon} \subseteq K, K$ being some fixed compact set of $\mathbb{R}^{N}$. Let us denote by $\hat{g}_{1}^{\varepsilon}$ the first Bloch coefficient defined by (3.12). We assume that $\hat{g}_{1}^{\varepsilon}$ is extended by zero outside $Y^{\prime} / \varepsilon$; then,

$$
\varepsilon^{N / 2} \hat{g}_{1}^{\varepsilon} \longrightarrow \mathcal{F}[g] \quad \text { weakly in } L_{\text {loc }}^{2}\left(\mathbb{R}^{N}\right), \text { as } \varepsilon \rightarrow 0 .
$$

Proof. For $\xi \in Y^{\prime} / \varepsilon$, we have

$$
\varepsilon^{N / 2} \hat{g}_{1}^{\varepsilon}(\xi)=\int_{\left(\mathbb{R}^{N}-\cup_{\mathbf{k}} \bar{T}_{\mathbf{k}}^{\varepsilon}\right)} g^{\varepsilon}(x) e^{-\mathbf{i} x \cdot \xi} \bar{\phi}_{1}^{\varepsilon}(x, \xi) d x=\int_{\mathbb{R}^{N}} \tilde{g}^{\varepsilon}(x) e^{-\mathbf{i} x \cdot \xi} \bar{\phi}_{1}\left(\frac{x}{\varepsilon}, \varepsilon \xi\right) d x .
$$

That is,

$$
\begin{aligned}
\varepsilon^{N / 2} \hat{g}_{1}^{\varepsilon}(\xi)= & \int_{\mathbb{R}^{N}} \tilde{g}^{\varepsilon}(x) e^{-\mathbf{i} x \cdot \xi} \bar{\phi}_{1}\left(\frac{x}{\varepsilon}, 0\right) d x \\
& +\int_{\mathbb{R}^{N}} \tilde{g}^{\varepsilon}(x) e^{-\mathbf{i} x \cdot \xi}\left[\bar{\phi}_{1}\left(\frac{x}{\varepsilon}, \varepsilon \xi\right)-\bar{\phi}_{1}\left(\frac{x}{\varepsilon}, 0\right)\right] d x
\end{aligned}
$$

On account of (3.19) and (3.20), the first integral in the right hand side of (3.33) is the Fourier transform of $\tilde{g}^{\varepsilon}, \mathcal{F}\left[\tilde{g}^{\varepsilon}\right](\xi)$, and it converges, when $\varepsilon \rightarrow 0$, towards 
the Fourier transform of $g$ weakly in $L^{2}\left(\mathbb{R}^{N}\right), \mathcal{F}[g](\xi)$. For small enough $\varepsilon$ and for $|\xi| \leq M$, the analyticity of $\phi_{1}(\cdot, \eta)$ in $\eta=0$ (see Theorem 3) allows us to conclude that

$$
\left\|\phi_{1}(\cdot, \varepsilon \xi)-\phi_{1}(\cdot, 0)\right\|_{L^{2}\left(Y^{*}\right)} \leq C \varepsilon .
$$

Taking into account that the number of $Y_{\mathrm{k}}$ contained in $K$ is of order $O\left(\varepsilon^{-N}\right)$, together with the $Y$-periodicity of $\bar{\phi}_{1}(\cdot, \eta)$ and the Cauchy Schwarz's inequality:

$$
\begin{aligned}
& \left|\int_{K} \tilde{g}^{\varepsilon}(x) e^{-\mathbf{i} x \cdot \xi}\left[\bar{\phi}_{1}\left(\frac{x}{\varepsilon}, \varepsilon \xi\right)-\bar{\phi}_{1}\left(\frac{x}{\varepsilon}, 0\right)\right] d x\right| \\
\leq & C\left\|\tilde{g}^{\varepsilon}\right\|_{L^{2}\left(\mathbb{R}^{N}\right)}\left\|\bar{\phi}_{1}(\cdot, \varepsilon \xi)-\bar{\phi}_{1}(\cdot, 0)\right\|_{L^{2}\left(Y^{*}\right)} \leq C \varepsilon .
\end{aligned}
$$

Hence, the second integral in the right hand side of (3.33) converges towards zero in $L_{\text {loc }}^{\infty}\left(\mathbb{R}^{N}\right)$.

Therefore, the convergence, as $\varepsilon \rightarrow 0$, of $\varepsilon^{N / 2} \hat{g}_{1}^{\varepsilon}(\xi)$ towards $\mathcal{F}[g](\xi)$ weakly in $L_{\text {loc }}^{2}\left(\mathbb{R}^{N}\right)$ holds and the proposition is proved.

4. Homogenization results. In this section we use the results in Section 3 concerning Bloch transform to derive convergence of the solution of problem (2.1) towards that of (2.4), when $\varepsilon \rightarrow 0$.

Throughout the section, for $g^{\varepsilon} \in L^{2}\left(\Omega^{\varepsilon}\right), \tilde{g}^{\varepsilon}$ will denote the extension by zero of $g^{\varepsilon}$ in the holes, while $P^{\varepsilon} u^{\varepsilon}$ is the extension of $u^{\varepsilon} \in H^{1}\left(\Omega^{\varepsilon}\right)$ to $H_{0}^{1}(\Omega)$ defined in $(2.3), \Omega^{\varepsilon}$ being $\left(\Omega-\bigcup_{\mathrm{k}=1}^{N(\varepsilon)} \bar{T}_{\mathrm{k}}^{\varepsilon}\right)$.

We observe that for $u^{\varepsilon}$, the solution of (2.2), there is a sequence still denoted by $u^{\varepsilon}$ such that

$$
\mid \begin{aligned}
& \tilde{u}^{\varepsilon} \longrightarrow u^{*} \text { weakly in } L^{2}(\Omega), \text { as } \varepsilon \rightarrow 0, \\
& P^{\varepsilon} u^{\varepsilon} \longrightarrow u^{0} \text { weakly in } H_{0}^{1}(\Omega) \text {, as } \varepsilon \rightarrow 0 .
\end{aligned}
$$

As a consequence of the localization process (see Theorem 4) we shall prove $u^{*}=u^{0}$. In fact, our aim here is to prove that $u^{0}$, as well as $u^{*}$, is the unique solution of the homogenized problem (2.4), so the whole sequence $\tilde{u}^{\varepsilon}$ converges towards $u^{0}=u^{*}$ weakly in $H_{0}^{1}(\Omega)$, as $\varepsilon \rightarrow 0$.

Let us first prove the convergence result formally: We assume that $\Omega=\mathbb{R}^{N}$ and that the conditions in Proposition 1 hold, and moreover that $\operatorname{supp} u^{\varepsilon} \subseteq K$, $K$ being a fixed compact in such a way that Proposition 3 can be applied.

That is to say, we assume $u^{\varepsilon} \in H^{1}\left(\mathbb{R}^{N}-\bigcup_{\mathrm{k}} \bar{T}_{\mathrm{k}}^{\varepsilon}\right)$ is the solution of

$$
A^{\varepsilon} u^{\varepsilon}=f \quad \text { in }\left(\mathbb{R}^{N}-\bigcup_{\mathrm{k}} \bar{T}_{\mathrm{k}}^{\varepsilon}\right)
$$


with a bounded gradient and $\operatorname{supp} u^{\varepsilon} \subseteq K$.

Taking into account formula (3.14) in Theorem 2, solving equation (4.2) is equivalent to solving

$$
\lambda_{m}^{\varepsilon}(\xi) \hat{u}_{m}^{\varepsilon}(\xi)=\hat{f}_{m}^{\varepsilon}(\xi), \quad \text { for all } m \geq 1 .
$$

Actually, thanks to Propositions 3 and (3.16), only the first Bloch coefficient has an influence on the limit problem. We study the asymptotic behavior of $\hat{u}_{1}^{\varepsilon}(\xi)$ by taking limits in the equation

$$
\lambda_{1}^{\varepsilon}(\xi) \hat{u}_{1}^{\varepsilon}(\xi)=\hat{f}_{1}^{\varepsilon}(\xi)
$$

Taking into account relations (3.9) and (3.10), we have

$$
\varepsilon^{N / 2} \hat{f}_{1}^{\varepsilon}(\xi)=\varepsilon^{N / 2} \hat{u}_{1}^{\varepsilon}(\xi) \frac{1}{\varepsilon^{2}} \lambda_{1}(\varepsilon \xi) .
$$

Theorem 3 allows us to write the Taylor expansion of $\lambda_{1}(\varepsilon \xi)$ for $|\xi| \leq M$ (for any fixed $M)$ and, in virtue of formulas (3.28), (3.29) and $\lambda_{1}(0)=0$, we have

$$
\frac{1}{\varepsilon^{2}} \lambda_{1}(\varepsilon \xi)=\frac{1}{2} \frac{\partial^{2} \lambda_{1}}{\partial \eta_{k} \partial \eta_{\ell}}(0) \xi_{k} \xi_{\ell}+o^{\varepsilon}(1)=a_{k \ell}^{h} \xi_{k} \xi_{\ell}+o^{\varepsilon}(1),
$$

where $\left|o^{\varepsilon}(1)\right| \leq C \varepsilon$ for $|\xi| \leq M$.

Multiplying (4.4) by $\varphi, \varphi \in \mathcal{D}\left(\mathbb{R}^{N}\right)$ with $\operatorname{supp} \varphi \subset K_{1}, K_{1}$ being any compact contained in $B(0, M)$, we have

$$
\begin{aligned}
\int_{\mathbb{R}^{N}} \varepsilon^{N / 2} \hat{f}_{1}^{\varepsilon}(\xi) \varphi(\xi) d \xi= & a_{k \ell}^{h} \int_{\mathbb{R}^{N}} \varepsilon^{N / 2} \hat{u}_{1}^{\varepsilon}(\xi) \xi_{k} \xi_{\ell} \varphi(\xi) d \xi \\
& +\int_{\mathbb{R}^{N}} o^{\varepsilon}(1) \varepsilon^{N / 2} \hat{u}_{1}^{\varepsilon}(\xi) \varphi(\xi) d \xi
\end{aligned}
$$

Let us consider $\tilde{f}^{\varepsilon}=f \chi_{\left(\mathbb{R}^{N}-\cup_{\mathrm{k}} \bar{T}_{\mathrm{k}}^{\varepsilon}\right)}$ where $\chi_{B}$ is the characteristic function of B. $\tilde{f}^{\varepsilon}$ takes the value $f$ on $\left(\mathbb{R}^{N}-\bigcup_{\mathrm{k}} \bar{T}_{\mathrm{k}}^{\varepsilon}\right)$ and zero outside. As is well known, when $\varepsilon \rightarrow 0, \tilde{f}^{\varepsilon}$ converges weakly in $L^{2}\left(\mathbb{R}^{N}\right)$ towards $f \theta$, where $\theta$ is the constant $\theta=\left|Y^{*}\right| /|Y|$. Thanks to Proposition 3, the limit, when $\varepsilon \rightarrow 0$, in (4.6) is

$$
\begin{aligned}
\int_{\mathbb{R}^{N}} \mathcal{F}[f \theta](\xi) \varphi(\xi) d \xi & =a_{k \ell}^{h} \int_{\mathbb{R}^{N}} \mathcal{F}\left[u^{*}\right](\xi) \xi_{k} \xi_{\ell} \varphi(\xi) d \xi \\
& =a_{k \ell}^{h} \int_{\mathbb{R}^{N}} \mathcal{F}\left[-\frac{\partial^{2} u^{*}}{\partial x_{k} \partial x_{\ell}}\right](\xi) \varphi(\xi) d \xi
\end{aligned}
$$

Therefore,

$$
-a_{k \ell}^{h} \frac{\partial^{2} u^{*}}{\partial x_{k} \partial x_{\ell}}=f \theta \quad \text { in } \mathcal{D}^{\prime}\left(\mathbb{R}^{N}\right)
$$


and we have proved that the solution $u^{\varepsilon}$ of (4.2) extended by zero in the holes converge towards the solution of the homogenized problem (4.7), as $\varepsilon \rightarrow 0$; that is the announced result when $\Omega=\mathbb{R}^{N}$.

We note that it is essential for the proof of (4.7) the fact that $u^{\varepsilon}$ belongs to the domain of definition of operator $A^{\varepsilon}$ (see Theorem 2).

Let us now extend the result to the general case in Section 2, where $\Omega$ is a bounded domain and $u^{\varepsilon}$ is the solution of (2.2). We always extend by zero outside $\Omega$.

Theorem 4. Let $f$ be a function of $L^{2}(\Omega)$. Let $u^{\varepsilon}$ be the solution of (2.2). Then, $P^{\varepsilon} u^{\varepsilon}$ converges weakly in $H_{0}^{1}(\Omega)$ towards $u^{0}$, as $\varepsilon \rightarrow 0$, where $u^{0}$ is the solution of (2.4).

Proof. The proof of the theorem is based on the equality

$$
\begin{gathered}
{\left[-a_{i j}^{h} \frac{\partial^{2} u^{*}}{\partial x_{i} \partial x_{j}}-f \theta\right] \phi+\left[-a_{i j}^{h} \frac{\partial u^{*}}{\partial x_{j}}+\chi_{i}^{*}\right] \frac{\partial \phi}{\partial x_{i}}-a_{i j}^{h} \frac{\partial\left(u^{*}-u^{0}\right)}{\partial x_{i}} \frac{\partial \phi}{\partial x_{j}}} \\
-\left[a_{i j}^{h}\left(u^{*}-u^{0}\right)\right] \frac{\partial^{2} \phi}{\partial x_{i} \partial x_{j}}=0 \quad \text { in } \mathcal{D}^{\prime}\left(\mathbb{R}^{N}\right),
\end{gathered}
$$

where $u^{*}$ and $u^{0}$ are the weak limits in $L^{2}(\Omega)$ and $H_{0}^{1}(\Omega)$ of $\tilde{u}^{\varepsilon}$ and $P^{\varepsilon} u^{\varepsilon}$ respectively, $\phi$ is any function $\phi \in \mathcal{D}(\Omega)$, and $\chi_{k}^{*}$ is the weak limit, as $\varepsilon \rightarrow 0$, of the extension by zero of $a_{k j}^{\varepsilon} \partial u^{\varepsilon} / \partial x_{j}$ inside the holes, i.e.,

$$
\widetilde{a_{k j}^{\varepsilon} \frac{\partial u^{\varepsilon}}{\partial x_{j}}} \rightarrow \chi_{k}^{*} \quad \text { weakly in } L^{2}(\Omega), \text { as } \varepsilon \rightarrow 0 .
$$

Let us assume that (4.8) holds for any $\phi \in \mathcal{D}(\Omega)$. Taking $\phi(x)=\phi_{0}(x) e^{\mathbf{i} n x \cdot \omega}$ in (4.8) for $\omega$ a unit vector in $\mathbb{R}^{N}, n \in \mathbb{N}$ and $\phi_{0} \in \mathcal{D}(\Omega)$, and then taking $\phi(x)=\phi_{0}(x) e^{-\mathbf{i} n x \cdot \omega}$, we subtract both expressions to obtain

$$
a_{i j}^{h}\left(u^{*}-u^{0}\right) \omega_{i} \omega_{j}=0 .
$$

Taking into account the ellipticity of matrix $a_{i j}^{h}$ we obtain $u^{0}=u^{*}$.

Therefore, (4.8) reads

$$
\left[-a_{k j}^{h} \frac{\partial^{2} u^{*}}{\partial x_{k} \partial x_{j}}-f \theta\right] \phi+\left[-a_{k j}^{h} \frac{\partial u^{*}}{\partial x_{k}}+\chi_{k}^{*}\right] \frac{\partial \phi}{\partial x_{j}}=0 .
$$

Then, the same argument for obtaining (4.10) (see also [11] for a similar argument) allows us to obtain

$$
-a_{k j}^{h} \frac{\partial u^{*}}{\partial x_{k}}=\chi_{k}^{*} \quad \text { and } \quad-a_{k j}^{h} \frac{\partial^{2} u^{*}}{\partial x_{k} \partial x_{j}}=f \theta \quad \text { in } \Omega,
$$


and, since $u^{*}=u^{0} \in H_{0}^{1}(\Omega)$, the result of Theorem 4 holds on the basis of (4.8).

Let us prove formula (4.8). To this end, we consider the solution $u^{\varepsilon}$ of $(2.2)$ and $\phi \in \mathcal{D}(\Omega)$. Clearly, $u^{\varepsilon} \phi \in H^{1}\left(\mathbb{R}^{N}-\bigcup_{\mathrm{k}} \bar{T}_{\mathrm{k}}^{\varepsilon}\right)$, supp $\left(u^{\varepsilon} \phi\right) \subset K, K$ being a fixed compact, and $\left\|u^{\varepsilon} \phi\right\|_{H^{1}\left(\mathbb{R}^{N}-\bigcup_{\mathrm{k}} \bar{T}_{\mathrm{k}}^{\varepsilon}\right)} \leq C$.

For each fixed $\varepsilon$, let $g$ denote the function $g(y)=u^{\varepsilon}(x) \phi(x)$. We consider the first Bloch transform of $g, \hat{g}_{1}(\eta)$. We use the fact that $\phi_{1}(\cdot, \eta)$ is an eigenfunction of (3.2) associated with $\lambda_{1}(\eta)$, that $g$ has a compact support, and that the equations (3.3) and (3.4) hold; then we integrate by parts to obtain:

$$
\begin{aligned}
\lambda_{1}(\eta) \hat{g}_{1}(\eta) & =\int_{\left(\mathbb{R}^{N}-\cup_{\mathbf{k}} \bar{T}_{\mathbf{k}}\right)} g(y) e^{-\mathbf{i} \eta \cdot y} \bar{\phi}_{1}(y, \eta) \lambda_{1}(\eta) d y \\
& =\int_{\left(\mathbb{R}^{N}-\cup_{\mathbf{k}} \bar{T}_{\mathbf{k}}\right)} g(y) \frac{\partial}{\partial y_{j}}\left[a_{i j} \frac{\partial}{\partial y_{i}}\left(\bar{\phi}_{1}(y, \eta) e^{-\mathbf{i} \eta \cdot y}\right)\right] d y \\
& =\int_{\left(\mathbb{R}^{N}-\cup_{\mathbf{k}} \bar{T}_{\mathrm{k}}\right)} \frac{\partial g}{\partial y_{j}} a_{i j}(y) \frac{\partial}{\partial y_{i}}\left[\bar{\phi}_{1}(y, \eta) e^{-\mathbf{i} \eta \cdot y}\right] d y .
\end{aligned}
$$

By introducing the change of variable $y=x / \varepsilon$ in (4.11), and taking into account the relations (3.9), (3.10), and (3.12), we obtain

$$
\begin{aligned}
\varepsilon^{N / 2}\left(\widehat{u^{\varepsilon} \phi}\right)_{1}^{\varepsilon}(\xi) \lambda_{1}^{\varepsilon}(\xi) & \int_{\left(\mathbb{R}^{N}-\cup_{\mathrm{k}} \bar{T}_{\mathrm{k}}^{\varepsilon}\right)} \frac{\partial}{\partial x_{j}}\left(u^{\varepsilon} \phi\right) a_{i j}^{\varepsilon}(x) \frac{\partial}{\partial x_{i}}\left[\bar{\phi}_{1}^{\varepsilon}(x, \xi) e^{-\mathbf{i} x \cdot \xi}\right] d x .
\end{aligned}
$$

Let us denote by $I$ the integral in the right hand side of (4.12); then we have

$$
\begin{aligned}
I= & \int_{\left(\mathbb{R}^{N}-\bigcup_{\mathrm{k}} \bar{T}_{\mathrm{k}}^{\varepsilon}\right)} \frac{\partial u^{\varepsilon}}{\partial x_{j}} a_{i j}^{\varepsilon} \frac{\partial}{\partial x_{i}}\left[\bar{\phi}_{1}^{\varepsilon}(x, \xi) e^{-\mathbf{i} x \cdot \xi} \phi\right] d x \\
& -\int_{\left(\mathbb{R}^{N}-\bigcup_{\mathrm{k}} \bar{T}_{\mathrm{k}}^{\varepsilon}\right)} \frac{\partial u^{\varepsilon}}{\partial x_{j}} a_{i j}^{\varepsilon} \frac{\partial \phi}{\partial x_{i}} \bar{\phi}_{1}^{\varepsilon}(x, \xi) e^{-\mathbf{i} x \cdot \xi} d x \\
& +\int_{\left(\mathbb{R}^{N}-\bigcup_{\mathrm{k}} \bar{T}_{\mathrm{k}}^{\varepsilon}\right)} \frac{\partial \phi}{\partial x_{j}} u^{\varepsilon} a_{i j}^{\varepsilon} \frac{\partial}{\partial x_{i}}\left(\bar{\phi}_{1}^{\varepsilon}(x, \xi) e^{-\mathbf{i} x \cdot \xi}\right) d x
\end{aligned}
$$

On account of (2.2), and since $\operatorname{supp} \phi \subset \Omega$,

$$
\begin{aligned}
I= & \int_{\left(\mathbb{R}^{N}-\bigcup_{\mathrm{k}} \bar{T}_{\mathrm{k}}^{\varepsilon}\right)} f \phi \bar{\phi}_{1}^{\varepsilon}(x, \xi) e^{-\mathbf{i} x \cdot \xi} d x \\
& -\int_{\left(\mathbb{R}^{N}-\bigcup_{\mathrm{k}} \bar{T}_{\mathrm{k}}^{\varepsilon}\right)} \frac{\partial u^{\varepsilon}}{\partial x_{j}} a_{i j}^{\varepsilon} \frac{\partial \phi}{\partial x_{i}} \bar{\phi}_{1}^{\varepsilon}(x, \xi) e^{-\mathbf{i} x \cdot \xi} d x \\
& -\mathbf{i} \xi_{i} \int_{\left(\mathbb{R}^{N}-\cup_{\mathrm{k}} \bar{T}_{\mathrm{k}}^{\varepsilon}\right)} \frac{\partial \phi}{\partial x_{j}} u^{\varepsilon} a_{i j}^{\varepsilon} \bar{\phi}_{1}^{\varepsilon}(x, \xi) e^{-\mathbf{i} x \cdot \xi} d x \\
& +\int_{\left(\mathbb{R}^{N}-\cup_{\mathrm{k}} \bar{T}_{\mathrm{k}}^{\varepsilon}\right)} \frac{\partial \phi}{\partial x_{j}} u^{\varepsilon} a_{i j}^{\varepsilon} \frac{\partial \bar{\phi}_{1}^{\varepsilon}}{\partial x_{i}}(x, \xi) e^{-\mathbf{i} x \cdot \xi} d x .
\end{aligned}
$$


We observe that the first three integrals appearing in (4.14) are the first coefficients of the Bloch transform of some functions in $L^{2}\left(\mathbb{R}^{N}-\bigcup_{\mathrm{k}} \bar{T}_{\mathrm{k}}^{\varepsilon}\right)$ whose support is contained in $\Omega$. We can apply Proposition 3 to calculate their limits, when $\varepsilon \rightarrow 0$.

Dealing with the fourth integral in (4.14), we take into account the smoothness of the first Bloch eigenfunction as stated in Theorem 3. We have, for $|\xi| \leq M$ ( $M$ fixed) and small enough $\varepsilon$, that

$$
\left\|\phi_{1}(\cdot, \varepsilon \xi)-\phi_{1}(\cdot, 0)-\xi_{k} \frac{\partial \phi_{1}}{\partial \eta_{k}}(\cdot, 0) \varepsilon\right\|_{H^{1}\left(Y^{*}\right)} \leq C \varepsilon^{2} .
$$

On account of (3.19) and (3.30), we have:

$$
\begin{aligned}
& \int_{\left(\mathbb{R}^{N}-\bigcup_{\mathrm{k}} \bar{T}_{\mathrm{k}}^{\varepsilon}\right)} \frac{\partial \phi}{\partial x_{j}} u^{\varepsilon} a_{i j}^{\varepsilon} \frac{\partial \bar{\phi}_{1}^{\varepsilon}}{\partial x_{i}}(x, \xi) e^{-\mathbf{i} x \cdot \xi} d x \\
& \quad=\xi_{k}(-\mathbf{i})|Y|^{-1 / 2} \int_{\left(\mathbb{R}^{N}-\bigcup_{\mathrm{k}} \bar{T}_{\mathrm{k}}^{\varepsilon}\right)} \frac{\partial \phi}{\partial x_{j}} u^{\varepsilon} a_{i j}^{\varepsilon}\left[\frac{\partial v^{k}}{\partial y_{i}}\left(\frac{x}{\varepsilon}\right)\right] e^{-\mathbf{i} x \cdot \xi} d x+o^{\varepsilon}(1)
\end{aligned}
$$

where $o^{\varepsilon}(1)$ means a function that converges weakly in $L_{\text {loc }}^{2}\left(\mathbb{R}^{N}\right)$ towards zero, as $\varepsilon \rightarrow 0$, and $v^{k}$ is the solution of (2.7). Therefore, from (4.13)-(4.15), (4.12) reads

$$
\begin{aligned}
& \varepsilon^{N / 2}\left(\widehat{u^{\varepsilon} \phi}\right)_{1}^{\varepsilon}(\xi) \lambda_{1}(\varepsilon \xi) \frac{1}{\varepsilon^{2}} \\
= & \varepsilon^{N / 2}(\widehat{f \phi})_{1}^{\varepsilon}(\xi)-\varepsilon^{N / 2}\left(\frac{\partial u^{\varepsilon}}{\partial x_{j}} a_{i j}^{\varepsilon} \frac{\partial \phi}{\partial x_{i}}\right)_{1}^{\varepsilon}(\xi)-\mathbf{i} \xi_{i} \varepsilon^{N / 2}\left(\widehat{u^{\varepsilon} a_{i j}^{\varepsilon} \frac{\partial \phi}{\partial x_{j}}}\right)_{1}^{\varepsilon}(\xi) \\
& -\mathbf{i} \xi_{k} \frac{1}{|Y|^{1 / 2}} \int_{\left(\mathbb{R}^{N}-\cup_{\mathrm{k}} \bar{T}_{\mathrm{k}}^{\varepsilon}\right)} u^{\varepsilon} \frac{\partial \phi}{\partial x_{j}} a_{i j}\left(\frac{x}{\varepsilon}\right) \frac{\partial v^{k}}{\partial y_{i}}\left(\frac{x}{\varepsilon}\right) e^{-\mathbf{i} x \cdot \xi} d x+o^{\varepsilon}(1) .
\end{aligned}
$$

In order to obtain the convergence of the left hand side of (4.16) as well as that of the first three terms in the right hand side, we apply Proposition 3 taking into account (4.5), the smoothness of $\phi$, the boundedness of $a_{i j}^{\varepsilon}$ and the following convergence, as $\varepsilon \rightarrow 0$ :

$$
\begin{array}{rlr}
\widetilde{f \chi}_{\left(\Omega-\bigcup_{\mathrm{k}} \bar{T}_{\mathrm{k}}^{\varepsilon}\right)} & \longrightarrow f \theta & \text { weakly in } L^{2}(\Omega), \\
\frac{\partial u^{\varepsilon}}{\partial x_{j}} a_{i j}^{\varepsilon} & \longrightarrow \chi_{i}^{*} & \text { weakly in } L^{2}(\Omega), \\
P^{\varepsilon} u^{\varepsilon} \tilde{a}_{i j}^{\varepsilon} & \longrightarrow u^{0} \mathcal{M}\left(\tilde{a}_{i j}\right) & \text { weakly in } L^{2}(\Omega),
\end{array}
$$

where $\chi_{i}^{*}$ is some element of $L^{2}(\Omega)$ and the extensions $\tilde{u}^{\varepsilon}$ and $P^{\varepsilon} u^{\varepsilon}$ are those in (4.1). Here $\mathcal{M}(\cdot)$ is the average in $Y$,

$$
\mathcal{M}(g)=\frac{1}{|Y|} \int_{Y} g d y \quad \text { for } g \in L^{2}(Y),
$$


and

$$
\mathcal{M}(\tilde{g})=\frac{1}{|Y|} \int_{Y-\bar{T}} g d y
$$

when $g$ is extended by zero on $\mathrm{T}$.

Finally, we observe that the last term in (4.16) is:

$$
\begin{aligned}
-\mathbf{i} \xi_{k} & \frac{1}{(2 \pi)^{N / 2}} \int_{\left(\mathbb{R}^{N}-\bigcup_{\mathrm{k}} \bar{T}_{\mathrm{k}}^{\varepsilon}\right)} u^{\varepsilon} \frac{\partial \phi}{\partial x_{j}} a_{i j}\left(\frac{x}{\varepsilon}\right) \frac{\partial v^{k}}{\partial y_{i}}\left(\frac{x}{\varepsilon}\right) e^{-\mathbf{i} x \cdot \xi} d x \\
= & -\mathbf{i} \xi_{k} \frac{1}{(2 \pi)^{N / 2}} \int_{\mathbb{R}^{N}} P^{\varepsilon} u^{\varepsilon} \frac{\partial \phi}{\partial x_{j}}\left[a_{i j}\left(\frac{x}{\varepsilon}\right) \frac{\partial w^{k}}{\partial y_{i}}\left(\frac{x}{\varepsilon}\right)\right] e^{-\mathbf{i} x \cdot \xi} d x \\
& +\mathbf{i} \xi_{k} \frac{1}{(2 \pi)^{N / 2}} \int_{\mathbb{R}^{N}} P^{\varepsilon} u^{\varepsilon} \frac{\partial \phi}{\partial x_{j}} \widetilde{a_{k j}\left(\frac{x}{\varepsilon}\right)} e^{-\mathbf{i} x \cdot \xi} d x .
\end{aligned}
$$

Now, thanks to the following convergence

$$
P^{\varepsilon} u^{\varepsilon} \longrightarrow u^{0} \quad \text { weakly in } H_{0}^{1}(\Omega) \text {, as } \varepsilon \rightarrow 0, \text { and }
$$

$$
a_{i j}\left(\frac{x}{\varepsilon}\right) \widetilde{\frac{\partial w^{k}}{\partial y_{i}}}\left(\frac{x}{\varepsilon}\right) \rightarrow \mathcal{M}\left(\widetilde{a_{i j} \frac{\partial w^{k}}{\partial y_{i}}}\right) \quad \text { weakly in } L^{2}(\Omega),
$$

we have the convergence of the integrals in the right hand side of (4.20) towards

$$
-\mathbf{i} \xi_{k} \mathcal{F}\left[u^{0} \frac{\partial \phi}{\partial x_{j}} \mathcal{M}\left(\widetilde{a_{i j}} \widetilde{\partial w^{k}} \frac{y_{i}}{\partial}\right)\right](\xi)+\mathbf{i} \xi_{k} \mathcal{F}\left[u^{0} \frac{\partial \phi}{\partial x_{j}} \mathcal{M}\left(\tilde{a}_{k j}\right)\right](\xi),
$$

weakly in $L_{\text {loc }}^{2}\left(\mathbb{R}^{N}\right)$.

On account of (4.17)-(4.23), we take weak limits in $L_{\text {loc }}^{2}\left(\mathbb{R}^{N}\right)$ in (4.16), as $\varepsilon \rightarrow 0$, and we use Fourier transform properties to obtain

$$
-a_{i j}^{h} \frac{\partial^{2}\left(u^{*} \phi\right)}{\partial x_{j} \partial x_{i}}=f \theta \phi-\chi_{i}^{*} \frac{\partial \phi}{\partial x_{i}}-\frac{\partial}{\partial x_{k}}\left(u^{0} \frac{\partial \phi}{\partial x_{j}} \mathcal{M}\left(\widetilde{a_{i j}} \widetilde{\partial w^{k}}\right)\right)
$$

in $\mathcal{D}^{\prime}\left(\mathbb{R}^{N}\right)$. We gather the terms containing $\phi$ and its derivatives in (4.24) and we get

$$
\begin{aligned}
{\left[-a_{i j}^{h} \frac{\partial^{2} u^{*}}{\partial x_{i} \partial x_{j}}-f \theta\right] \phi=\left[-\chi_{i}^{*}+a_{i j}^{h} \frac{\partial u^{*}}{\partial x_{j}}\right] \frac{\partial \phi}{\partial x_{i}} } \\
+\left[a_{j k}^{h} \frac{\partial u^{*}}{\partial x_{k}}-\frac{\partial u^{0}}{\partial x_{k}} \mathcal{M}\left(\widetilde{a_{i j} \frac{\partial w^{k}}{\partial y_{i}}}\right)\right] \frac{\partial \phi}{\partial x_{j}} \\
+\left[u^{*} a_{k j}^{h}-\mathcal{M}\left(\widetilde{a_{i j} \frac{\partial w^{k}}{\partial y_{i}}}\right) u^{0}\right] \frac{\partial^{2} \phi}{\partial x_{k} \partial x_{j}} .
\end{aligned}
$$


Formula (2.8) allows us to conclude

$$
\mathcal{M}\left(\widetilde{a_{i j} \frac{\partial w^{k}}{\partial y_{i}}}\right)=a_{j k}^{h},
$$

and hence (4.8) holds. This completes the proof of Theorem 4.

Remark 2. Note that, in the proof of Theorem 4, we obtain that the function $\tilde{u}^{\varepsilon}$, extension by zero of $u^{\varepsilon}$ in the holes, converges weakly in $L^{2}(\Omega)$ towards $u^{0}$, as $\varepsilon \rightarrow 0$, where $u^{0}$ is the solution of the homogenized problem.

Remark 3. We observe that the assumption made about the geometry of the domain $\Omega$ and the holes in Section 2 can be weakened. For example, if the holes meet the boundary of $\Omega$ and the Dirichlet condition is imposed on $\Gamma^{\varepsilon}=\partial \Omega-\cup T^{\varepsilon}$, the proof in Theorem 4 still holds: we only require the existence of a uniformly bounded family of extension operators $P^{\varepsilon}$ and the bound for the solutions $\left\|u^{\varepsilon}\right\|_{H^{1}\left(\Omega^{\varepsilon}\right)} \leq C$, with $C$ a constant independent of $\varepsilon$ (see [18], [19] Section I.4, and [4]). We can also consider more general geometries for the holes, provided that $\Omega^{\varepsilon}$ is a connected open bounded domain with a Lipschitz boundary and $d(\bar{T}, \partial Y)>0$. See [1] for a different proof of the convergence using a suitable extension operator, as well as for more general geometries of the domain $\Omega^{\varepsilon}$.

Acknowledgment. The authors would like to thank the anonymous referee for several sharp comments which enabled them to improve and revise the original version.

\section{REFERENCES}

[1] E. Acerbi, V. Chiado-Piat, G. D. Maso $\mathcal{E}$ D. Percivale, An extension theorem for connected sets, and homogenization in general periodic domains, Nonlinear Anal., 18 (1992), 481-496.

[2] G. Allaire 83 C. Conca, Bloch wave homogenization for a spectral problem in fluid-solid structures, Arch. Rational. Mech. Anal., 135 (1996), 197-257.

[3] _ Boundary layers in the homogenization of a spectral problem in fluid-solid structures, Siam J. Math. Anal., 29 (1998), 343-379.

[4] G. Allaire 83 F. Murat, Homogenization of the Neumann problem with nonisolated holes, Asymptotic Anal., 7 (1993), 81-95.

[5] H. Aтtouch, Variational Convergence for Functions and Operators, In: Applied Mathematics, 8, Pitman, London, 1984. 
[6] A. Bensoussan, J. L. Lions \& G. Papanicolaou, Asymptotic Analysis for Periodic Structures, North-Holland, Amsterdam, 1978.

[7] F. BLOCH, Über die quantenmechanik der electronen in kristallgittern, Z. Phys., 52 (1928), 555-600.

[8] D. Cioranescu \& J. S. J. Paulin, Homogenization in open sets with holes, J. Math. Anal. Appl., 71 (1979), 590-607.

[9] C. Conca \& P. Donato, Non homogeneous Neumann problems in domains with small holes, Rairo Modél. Math. Anal. Numér., 22 (1998), 561-607.

[10] C. Conca, J. Planchard \& M. Vanninathan, Fluids and Periodic Structures, In: Research in Applied Mathematics, 38, J. Wiley/Masson, New York-Paris, 1995.

[11] C. Conca $\&$ M. Vanninathan, Homogenization of periodic structures via Bloch decomposition, Siam J. Appl. Math., 57 (1997), 1639-1659.

[12] _ _ On uniform $H^{2}$-estimates in periodic homogenization, Proc. Roy. Soc. Edinburgh Sect. A (1999); submitted paper.

[13] G. Dal Maso, An Introduction to $\Gamma$-Convergence. Progress in Nonlinear Differential Equations and Their Applications, 8, Birkhäuser, Boston, 1993.

[14] E. De Giorgi \& S. Spagnolo, Sulla convergenza degli integrali dell'energia per operatori ellittici del secondo ordine, Boll. UMI, 8 (1973), 391-411.

[15] L. Hörmander, An Introduction to Complex Analysis in Several Variables, North-Holland, Amsterdam, 1973.

[16] T. KAто, Perturbation Theory for Linear Operators, Springer-Verlag, Berlin, 1966.

[17] F. Murat 6 L. Tartar, H-convergence, In: Topics in the Mathematical Modeling of Composite Materials, (A. Cherkaev et al. eds.), Progress in Nonlinear Differential Equations and their Applications, Boston, Birkhäuser, 1997, pp. 2144.

[18] O. A. Oleinik, A. S. Shamaev, E G. A. Yosifian, The Neumann problem for second order elliptic equations with rapidly oscillating periodic coefficients in a perforated domain, In: Partial Differential Equations and the Calculus of Variations. Essays in Honor of Ennio De Giorgi, (F. Colombini et al. eds.), vol. II, Boston, Birkhäuser, 1989, pp. 879-904.

[19] _ Mathematical Problems in Elasticity and Homogenization, North-Holland, Amsterdam, 1992.

[20] O. A. Oleinik, T. Shaposhnikova, On homogenization problems for the Laplace operator in partially perforated domains with Neumann's condition on the boundary of cavities, Rend. Mat. Acc. Lincei, s.6, v.6, 1995, pp. 133-142. 
[21] J. Sanchez-Hubert $\&$ E. Sanchez-Palencia, Vibration and Coupling of Continuous Systems. Asymptotic Methods, Springer-Verlag, Heidelberg, 1989.

[22] J. Sanchez-Hubert $\mathscr{E}$ N. Turbé, Ondes élastiques dans une bande périodique, Rairo Modél. Math. Anal. Numér., 20 (1986), 539-561.

[23] E. Sanchez-Palencia, Non-Homogeneous Media and Vibration Theory, In: Lecture Notes in Physics, 127, Springer-Verlag, Berlin, 1980.

[24] F. Santosa, A dispersive effective medium theory for waves in periodic media, In: Mathematical and Numerical Aspects of Wave Propagation Phenomena, (G. Cohen et al. eds.), Philadelphia, 1991, Siam, pp. 671-679.

[25] F. Santosa $\mathscr{E} \mathrm{W}$. W. Symes, A dispersive effective medium for wave propagation in periodic composites, Siam J. Appl. Math., 51 (1991), 984-1005.

[26] S. Spagnolo, Sulla convergenza di soluzioni di equazioni paraboliche ed ellittiche, Ann. Sc. Norm. Sup. Pisa, 22 (1968), 571-597.

[27] C. Wilcox, Theory of Bloch waves, J. Anal. Math., 33 (1978), 146-167.

C. CONCA

Departamento de Ingeniería Matemática

Facultad de Ciencias Físicas y Matemáticas

Universidad de Chile, Santiago, CHILE

EMAIL: cconca@dim.uchile.cl

D. Gómez, M. Lobo, M. E. Pérez

Departamentos de Matemáticas

Universidad de Cantabria

Santander, ESPAÑA

EMAIL: meperez@besaya.unican.es

KEYWORDS: homogenization, Bloch waves, spectral perturbation.

Submitted: Mach 24th, 1999, revised: July 27th, 1999. 